\title{
Atividade física e dor lombar em brasileiros: uma revisão sistemática
}

\author{
Physical activity and low back pain in Brazilians: a systematic review
}

\section{AUTORES \\ Gabriela Ribeiro Polli ${ }^{1}$ (D) \\ Hugo Falqueto ${ }^{2}$ (D) \\ Izadora Czarnobai ${ }^{2}$ (iD \\ Diego Giulliano Destro Christofaro ${ }^{3}$ \\ Paulo Henrique Guerra ${ }^{1,2}$ (D) \\ 1 Grupo de Estudos e Pesquisas Epidemiológicas em Atividade Física e Saúde da Universidade de São Paulo, São Paulo, São Paulo, Brasil. \\ 2 Universidade Federal da Fronteira Sul, Chapecó, Santa Catarina, Brasil. \\ 3 Universidade Estadual de São Paulo, Departamento de Educação Física, Presidente Prudente, São Paulo, Brasil.}

\section{CONTATO}

Gabriela Ribeiro Polli

ft_gabiribeiro@hotmail.com

Avenida Osmundo dos Santos Pelegrini, 1460, Apartamento 04, Bloco D, Jardim do

Trevo, Jundiaí, São Paulo, Brasil.

CEP: 13211-377.

DOI

$10.12820 /$ rbafs.23e0047

\section{(cc) BY-NC-SA}

Este obra está licenciado com uma Licença Creative Commons Atribuição-NãoComercialCompartilhaIgual 4.0 Internacional.

\begin{abstract}
RESUMO
O objetivo deste estudo é identificar e sumarizar os estudos observacionais conduzidos em território brasileiro que avaliaram as possíveis associações entre a prática de atividade física (AF) e a dor lombar (DL), bem como analisar seus métodos. Para isto, foram realizadas buscas em cinco bases de dados eletrônicas (Lilacs, Pubmed, Scielo, Sportdiscus e Web of Science), além de buscas manuais nas listas de referências. Foram incluídos estudos observacionais desenvolvidos em território brasileiro, sem restrições quanto à idade, que apresentaram análises de associações entre $\mathrm{AF}$ e $\mathrm{DL}$, disponíveis até 10 de outubro de 2017. Dos 153 estudos inicialmente recuperados, 11 compuseram a síntese descritiva (sete deles envolvendo adultos). Das 14 análises de associação recuperadas, apenas uma apresentou associação de risco entre a prática excessiva de AF e DL, em adolescentes. A presente síntese aponta para a necessidade de importantes avanços metodológicos nos futuros estudos, tais como: a necessidade da realização de estudos longitudinais; a investigação de possíveis relações entre distintos domínios da $\mathrm{AF}$ e diferentes tipos de DL; a necessidade de padronização nos pontos de corte utilizados pelos instrumentos para determinar AF suficiente e a prevalência da DL; e a utilização de medidas objetivas, em paralelo ao questionário, para avaliação da AF.
\end{abstract}

Palavras-chave: Atividade física; Dor lombar; Adolescentes; Adultos; Brasil; Revisão.

\begin{abstract}
The objective of this study was to identify and summarize the available studies conduced in Brazil, which evaluated possible associations between physical activity (PA) and low back pain (LBP), as well as to analyse their methods. Search was performed in five electronic databases (Lilacs, Pubmed, Scielo, Sportdiscus and Web of Science) and by manual searches in reference lists. Inclusion criteria were: observational studies available until October 10, 2017; which presented association analyses between PA and LBP; sample of Brazilian individuals, with no restrictions about age Of the 153 initially studies recovered, 11 were included (seven of them involving adults). Of the 14 analyses recovered, only one association was observed between excess of PA and LBP in adolescents. Most of the included articles did not show significant associations between $P A$ and LBP. Important methodological advances are needed in future studies on this thematic, such as: longitudinal studies; the investigation of possible relationships between $L B P$ and the distinct domains of $P A$; standardization in the cut-off points used to determine the prevalence of $L B P$ and sufficient $P A$; the use of objective measures, in parallel to the questionnaire, for the PA assessment.
\end{abstract}

Keywords: Physical activity; Low back pain; Adolescents; Adults; Brazil; Review.

\section{Introdução}

$\mathrm{Na}$ literatura, são bem reconhecidos os diversos benefícios da prática de atividade física (AF) regular nas distintas fases da vida ${ }^{1}$. Deste modo, ao passo que se atribui à $\mathrm{AF}$ um papel de destaque na promoção de saúde, na prevenção de diversas doenças/agravos à saúde, na melhora da capacidade motora geral, da dor crônica e das desordens psicológicas e mentais ${ }^{2-3}$, também se reconhece que sua ausência está associada à inúmeros danos à saúde, entre eles o desconforto musculoesquelético ${ }^{4}$.

Dentre esses desfechos de saúde, a dor lombar (DL) é um desconforto musculoesquelético que apresenta elevada prevalência e pode afetar de forma significativa a qualidade de vida das pessoas ${ }^{5-6}$. Estudos prévios sugerem que a maior parcela da população sofrerá sintomas de DL em algum momento da vida ${ }^{6-7}$. Além disso, a literatura também aponta para a dificuldade de se fazer seu diagnóstico e orientação para tratamento ${ }^{8}$.

Apesar da incidência da DL aumentar com a idade, ela é mais prevalente entre adultos na faixa dos 40 aos 69 anos. Todavia, é reconhecido que seus primeiros sintomas aparecem no período da pré-adolescência, 
tornando aumentado o risco de se desenvolvê-la na vida adulta $^{10-11}$. A DL está enquadrada como uma das principais causas de incapacidade no mundo ${ }^{5-6}$ e a responsável pela maior quantidade de anos vividos com incapacidade, dentre todas as outras condições de saúde ${ }^{12}$. No Brasil, o quadro não é diferente, sendo a dor nas costas a principal causa de aposentadoria por invalide $z^{13}$.

Dessa forma, do ponto de vista da saúde pública, é importante o reconhecimento dos fatores que estão associados à $\mathrm{DL}^{14}$. Duas revisões prévias sugerem que a prática de $\mathrm{AF}$ é uma importante estratégia para a prevenção e/ou controle da DL ${ }^{15-16}$. Contudo, mesmo que as sínteses destas revisões estejam baseadas em evidências de estudos de coorte ${ }^{15}$ e estudos de intervenção ${ }^{16}$, em ambas, não foram incluídas pesquisas conduzidas no território brasileiro.

O reconhecimento do estado da arte dos estudos conduzidos no Brasil justifica-se tanto pela identificação das evidências disponíveis sobre as possíveis associações entre as variáveis, quanto pela possibilidade de estudo e análise dos seus aspectos metodológicos, de forma que a evidência produzida possa fortalecer a tomada de decisão e apoiar futuros estudos na temática. Nesse sentido, o presente estudo teve como objetivo identificar e sumarizar os estudos observacionais conduzidos em território brasileiro que avaliaram as possíveis associações entre a prática de atividade física (AF) e a dor lombar (DL) nas distintas fases da vida, bem como discutir seus métodos.

\section{Método}

O presente estudo configura-se como uma revisão sistemática da literatura. Não houve registro prévio do seu protocolo. Como critérios de inclusão, estipulou-se previamente que a síntese seria composta por artigos: 1) com delineamentos observacionais (por exemplo: transversais, casos-controles e coortes); 2) que envolveram indivíduos que vivem no território brasileiro, independentemente da fase da vida das condições de saúde, e da representatividade da amostra (local, regional, nacional); 3) que apresentassem análises de associações entre a AF e DL, baseadas em modelos de regressão, sem distinções em relação ao uso das variáveis enquanto exposição e desfecho.

Diante das particularidades entre os delineamentos de pesquisa e das limitações em sua comparação, desde os primeiros passos da revisão não se pretendeu envolver estudos de intervenção.

Por outro lado, diante do intuito de apresentar o estado da arte da literatura brasileira na temática, não foram colocadas restrições quanto aos domínios da $\mathrm{AF}$ pesquisados (por exemplo: lazer, deslocamento, ocupacional e doméstico, assim como exercícios físicos como dimensões específicas da AF), assim como em relação às definições e formas de avaliação da DL.

Para recuperação da evidência, no dia 10 de outubro de 2017, buscas sistemáticas foram aplicadas em cinco bases de dados eletrônicas (Lilacs, Pubmed, Scielo, Sportdiscus e Web of Science), partindo dos termos e combinações desenvolvidas e utilizadas no Pubmed: (low back pain[Text Word]) OR lumbago[Text Word]) OR backache[Text Word]) OR sciatica[Text Word]) AND (physical activity[Text Word]) OR exercise[Text Word]) AND Brazil[Text Word]). Para evitar a perda de dados relevantes, também foram conduzidas buscas manuais nas listas de referências dos artigos avaliados pelos seus textos integrais. Não foram aplicadas restrições em relação ao ano de publicação dos estudos.

A avaliação por títulos e resumos e pelos textos integrais foi conduzida por dois pesquisadores independentes (HF e IC), com auxílio de um terceiro pesquisador, sênior, para diminuir dúvidas e estabelecer consenso (PG).

A extração dos dados dos estudos originais também foi realizada por dois pesquisadores (GP e PG), de forma independente, dividindo-se a informação em três domínios: I) dados descritivos dos artigos originais (por exemplo: local da pesquisa, ano da coleta, tamanho da amostra, percentual de mulheres na amostra, faixa etária e média de idade); II) aspectos metodológicos dos artigos (por exemplo: delineamento de estudo, método utilizado para amostragem, definição de AF e seus respectivos pontos de corte, instrumento de avaliação da AF, instrumento utilizado para avaliação da DL, período do instrumento para determinação da prevalência de DL e prevalência de DL na amostra) e III) resultados, classificando-os por categorias de exposição, desfecho, grupos de referência, métodos utilizados nas análises, variáveis ajustadas, magnitude dos dados analisados e sua respectiva classificação (por exemplo: a AF como fator de risco, fator de proteção, ou dados estatisticamente nulos). Considerando-se a experiência prévia de alta heterogeneidade metodológica nas sínteses baseadas em evidências de estudos observacionais, não se planejou a elaboração de meta-análise. Não obstante, devido ao caráter descritivo da presente revisão, não foi avaliado o risco de viés/qualidade metodológica de cada estudo. 


\section{Resultados}

Após a identificação e exclusão dos registros duplicados entre as bases de dados e os estudos de revisão $(n=34)$, 119 artigos potenciais foram avaliados por seus títulos e resumos (Figura 1). Destes, 29 foram encaminhados para a avaliação pelos seus textos integrais, onde 18 deles foram excluídos, tendo como principais motivos: a inexistência de dados específicos de dor lombar $(\mathrm{n}=$ 6); e a inexistência de análises de associação entre AF e DL ( $\mathrm{n}=5$ ). Dessa forma, a síntese descritiva da presente revisão foi composta por 11 artigos originais ${ }^{17-27}$.

No geral, todos os estudos analisados apresentaram o delineamento transversal. Por região do país, a maior

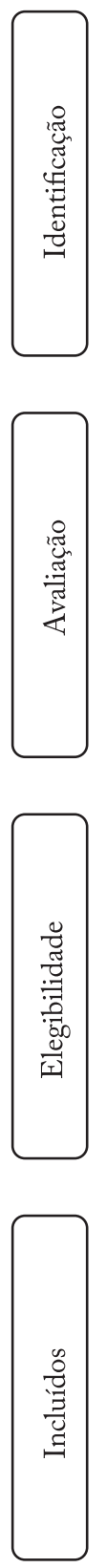

Estudos identificados pelas buscas nas bases de dados eletrônicas

$$
(\mathrm{n}=150)
$$

parte dos estudos foi conduzida nas regiões $\mathrm{Sul}(\mathrm{n}=$ $5)^{18,19,23,24,26}$ e Sudeste $(n=4)^{17,22,25,27}$ (Tabela 1$)$. Sete estudos utilizaram-se de técnicas aleatórias para a seleção das suas amostras ${ }^{19,21,23-27}$, sendo que as amostras variaram entre $163^{25}$ e 3.182 participantes ${ }^{26}$, com maior percentual do sexo feminino em 8 deles $(78,5 \%)^{17,19-21,23,24-27}$.

No conjunto de estudos direcionados às crianças e adolescentes, dois consideraram a AF de $\operatorname{Lazer}^{17,18}$, tendo utilizado questionários próprios para a sua medida (Tabela 2). A respeito dos pontos de corte utilizados para classificação da AF suficiente, dois estudos direcionados às crianças e adolescentes não descreveram os pontos de corte utilizados ${ }^{17,20}$. Em adultos,

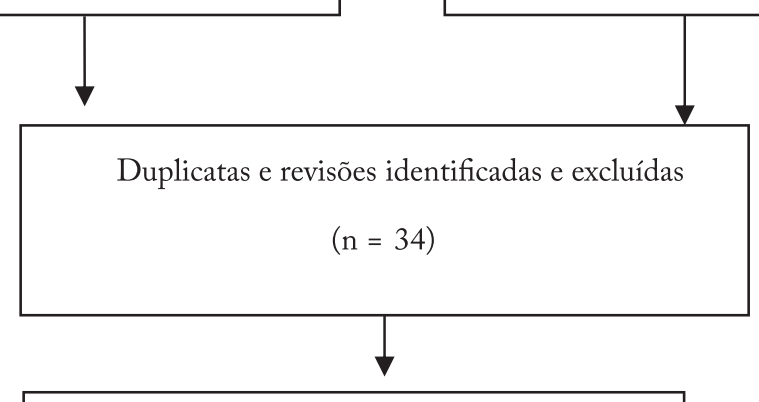

Referências avaliadas por títulos e resumos

$$
(\mathrm{n}=119)
$$

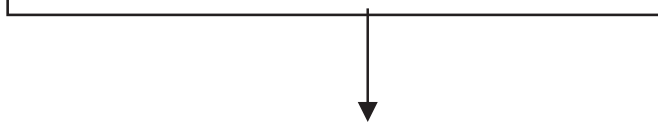

Avaliação por texto integral / Extração de dados

$(\mathrm{n}=29)$

Síntese descritiva $(\mathrm{n}=11)$

Crianças e adolescentes $(n=4)$;

Adultos $(\mathrm{n}=7)$
Referências excluídas

$$
(\mathrm{n}=90)
$$

Figura 1 - Fluxograma de seleção dos estudos da revisão sistemática. 
quatro estudos ${ }^{21,23,25,26}$ consideraram todos os domínios da AF. Em quatro estudos observou-se a utilização de versões do International Physical Activity Questionnaire $\left(\right.$ IPAQ ${ }^{23-26}$. Sobre pontos de corte para classificação de AF suficiente, três estudos consideraram 150 minutos semanais de AF moderadas e vigorosas $23,26,14,26$ e dois consideraram 180 minutos semanais de AF moderadas e vigorosas ${ }^{21,27}$.

Em adolescentes, quatro instrumentos foram utilizados para avaliação da DL, sendo um deles um questionário próprio ${ }^{19}$ e em outro, uma adaptação do Questionário Nórdico ${ }^{17}$. Em adultos, quatro estudos

Tabela 1 - Dados descritivos dos artigos incluídos na revisão sistemática sobre a relação entre atividade física e dor lombar ( $\mathrm{n}=11)$.

\begin{tabular}{|c|c|c|c|c|c|}
\hline Referências & Local/Ano da coleta de dados & $\begin{array}{l}\text { Técnica utilizada para } \\
\text { composição da amostra (n) }\end{array}$ & $\begin{array}{c}\text { Faixa etária } \\
\text { (média de idade) }\end{array}$ & $\% \mathrm{~F}$ & Características da amostra \\
\hline \multicolumn{6}{|l|}{ Crianças e adolescentes } \\
\hline De Vitta et al. ${ }^{17}$ & Bauru (SP)/2007 & $\mathrm{C}(1.236)$ & $11-14(14)$ & 52 & Estudantes de escolas públicas \\
\hline Lemos et al. ${ }^{18}$ & Porto Alegre (RS)/nd & $\mathrm{C}(770)$ & $7-17$ (nd) & 50 & Estudantes de escola privada \\
\hline Onofrio et al. ${ }^{19}$ & Pelotas (RS)/2009 & $\mathrm{A}(1.233)$ & $13-19(16)$ & 54 & $\begin{array}{c}\text { Estudantes de escolas públicas } \\
\text { e privadas }\end{array}$ \\
\hline Silva et al. ${ }^{20}$ & Recife $(\mathrm{PE}) / \mathrm{nd}$ & $\mathrm{C}(961)$ & $14-19(17)$ & 61 & Estudantes da rede pública \\
\hline \multicolumn{6}{|l|}{ Adultos } \\
\hline Almeida et al. ${ }^{21}$ & Salvador $(\mathrm{BA}) / 2000$ & $\mathrm{~A}(2.297)$ & $20-94(41)$ & 56 & \\
\hline Andrusaitis et al. ${ }^{22}$ & Estado de São Paulo/2003 & $\mathrm{C}(410)$ & $20-69(40)$ & nd & $\begin{array}{l}\text { Caminhoneiros com mais de um } \\
\text { ano de experiência profissional }\end{array}$ \\
\hline Ferreira et al. ${ }^{23}$ & Pelotas $(\mathrm{RS}) / \mathrm{nd}$ & A (972) & $20-69(41)$ & 57 & - \\
\hline Meucci et al. ${ }^{24(\mathrm{a})}$ & Pelotas (RS)/2010 & $\mathrm{A}(2.732)$ & $20-70(\mathrm{nd})$ & 58 & - \\
\hline Santana et al. ${ }^{25}$ & $\begin{array}{l}\text { Ouro Preto, Mariana e João } \\
\text { Monlevade (MG)/2013-15 }\end{array}$ & $A(163)$ & nd & 34 & Professores universitários \\
\hline Silva et al. ${ }^{26}$ & Pelotas (RS)/2002 & $\mathrm{A}(3.182)$ & $20-70(44)$ & 57 & - \\
\hline Zanuto et al. ${ }^{27}$ & Presidente Prudente (SP)/nd & $\mathrm{A}(743)$ & $>18$ anos & 61 & $\begin{array}{l}\text { Moradores por mais de dois } \\
\text { anos na zona urbana. }\end{array}$ \\
\hline
\end{tabular}

$\% \mathrm{~F}$ = percentual de mulheres na amostra; $(\mathrm{a})$ = foram considerados válidos apenas os dados da segunda coleta (a primeira coleta está descrita no estudo de Silva et $\left.a{ }^{26}{ }^{26}\right) ; \mathrm{A}=$ amostra composta por técnicas aleatórias; $\mathrm{C}=$ amostragem por conveniência; nd = não descrito.

Tabela 2 - Síntese dos aspectos metodológicos dos artigos incluídos na revisão sistemática sobre a relação entre atividade física e dor lombar $(\mathrm{n}=11)$.

\begin{tabular}{|c|c|c|}
\hline Referências & $\begin{array}{l}\text { Atividade física: Domínio(s) considerado (s)/Método de } \\
\text { avaliação (pontos de corte) }\end{array}$ & Dor lombar: método de avaliação/prevalência \\
\hline \multicolumn{3}{|l|}{ Crianças e adolescentes } \\
\hline De Vitta et al. ${ }^{17}$ & Lazer/QDE (nd) & Questionário Nórdico adaptado/19,5\% nos últimos 12 meses $^{(\mathrm{b})}$ \\
\hline Lemos et al. ${ }^{18}$ & Lazer/QDE (120 min/sem) & Questionário adaptado de Vidal et al. ${ }^{(\mathrm{c})} / 31,6 \%$ no último mês ${ }^{(\mathrm{b})}$ \\
\hline Onofrio et al. ${ }^{19}$ & $\begin{array}{l}\text { nd/Questionário utilizado em Bastos et al. }{ }^{(a)} \text { (300 min/sem AF } \\
\text { moderadas e vigorosas) }\end{array}$ & QDE/13,7\% no último mês ${ }^{(\mathrm{b})}$ \\
\hline Silva et al. ${ }^{20}$ & nd/IPAQ Curto (nd) & Diagrama corporal/30,5\% nos últimos seis meses ${ }^{(\mathrm{e})}$ \\
\hline \multicolumn{3}{|l|}{ Adultos } \\
\hline Almeida et al. ${ }^{21}$ & $\begin{array}{l}\text { Todos os domínios/QDE (180 min/sem AF moderadas e } \\
\text { vigorosas) }\end{array}$ & QDE/14,7\% nos últimos seis meses ${ }^{(\mathrm{e})}$ \\
\hline Andrusaitis et al. ${ }^{22}$ & nd/QDE (nd) & QDE/59\% durante o tempo profissional ${ }^{(f)}$ \\
\hline Ferreira et al. ${ }^{23}$ & $\begin{array}{l}\text { Todos os domínios, com estratificação para atividade física de } \\
\text { Lazer/IPAQLongo ( } 150 \mathrm{~min} / \mathrm{sem})\end{array}$ & QDE/25,2\% nos últimos 12 meses $^{(\mathrm{b})}$ \\
\hline Meucci et al. ${ }^{24}$ & Lazer/IPAQ Curto (150 min/sem) & $\begin{array}{l}\text { Questionário Nórdico adaptado/ 9,6\% apresentou dor lombar } \\
\text { com duração } \geq \text { que } 7 \text { semanas nos últimos } 3 \text { meses }^{(\mathrm{e})}\end{array}$ \\
\hline Santana et al. ${ }^{25}$ & Todos os domínios/IPAQCurto (450 met/min/sem) & QDE/33\% durante toda a vida ${ }^{(f)}$ \\
\hline Silva et al. ${ }^{26}$ & Todos os domínios/IPAQ Curto (150 min/sem) & $\begin{array}{l}\text { Questionário Nórdico adaptado/4,2\% com duração } \geq 7 \\
\text { semanas }^{(e)}\end{array}$ \\
\hline Zanuto et al. ${ }^{27}$ & $\begin{array}{l}\text { Lazer/Questionário de Baecke (180 min/sem AF moderadas } \\
\text { e vigorosas) }\end{array}$ & $\begin{array}{l}\text { Questionário Kuorinka et al. }{ }^{(\mathrm{h})} / 50,2 \% \text { nos últimos } 12 \text { meses e } \\
\text { também na última semana }{ }^{(\mathrm{b})}\end{array}$ \\
\hline
\end{tabular}

(a) = Bastos et al. ${ }^{40}(\mathrm{~b})=$ Sem classificação de dor lombar crônica ou aguda; $(\mathrm{c})=\operatorname{Vidal}^{41}(\mathrm{~d})=18,2 \%$ Dor crônica por mais de 3 meses, e $28,6 \%$ dor aguda no último mês; (e) = Dor crônica; (f) = 41\% dor ocasional e $18 \%$ dor constante; $(\mathrm{g})$ = Dor lombar crônica diagnosticada por um médico; (h) = Kuorinka al. ${ }^{42}$; min/sem: minutos por semana; nd: não descrito; QDE = questionário desenvolvido para o estudo. 
utilizaram questionários próprios para avaliação da $\mathrm{DL}^{21-23,25}$ e em dois houve a aplicação de versões adaptadas do Questionário Nórdico ${ }^{24,26}$. De forma geral, os períodos dos instrumentos para determinação das prevalências de DL também apresentaram grande variação entre os estudos, independente da faixa etária, o que impossibilitou a comparação entre as prevalências (Tabela 2).

Considerando o conjunto de estudos incluídos, em seis não se identificou a classificação de DL (se aguda e/ou crônica) ${ }^{17-20,23,27}$. Em adultos, quatro estudos avaliaram apenas a presença de DL crônica ${ }^{21,24-26} \mathrm{e}$, em outro, avaliou-se tanto a presença de DL aguda como crônica ${ }^{22}$. Complementarmente, os critérios adotados para as classificações da DL foram muito distintos entre as pesquisas (Tabela 2).

$\mathrm{Na}$ síntese de resultados (Tabela 3), ao todo, foram encontradas 14 análises que investigaram possíveis associações entre a prática de AF e DL, sendo nove delas em artigos que envolveram populações adultas (60\%). Foram observadas distintas técnicas de regressão utilizadas para as análises, assim como em relação as variáveis ajustadas e grupos de referência.

Nas pesquisas conduzidas com crianças e adolescentes, um dado pode ser destacado: chance aumentada de DL no grupo dos adolescentes envolvidos em práticas esportivas competitivas fora da escola $(\mathrm{OR}=$ 2,58; IC95\%: 1,29-3,48) $)^{17}$. Em adultos, nenhum estudo apresentou associação estatisticamente significativa (nem para risco, nem para proteção) entre as variáveis de interesse. Em vista do elevado número de análises com resultados nulos, não foram observadas diferenças entre os estudos que fizeram ajuste mínimo e os que não fizeram.

\section{Discussão}

Os achados desta revisão sistemática fundamentaram-se nos dados de 11 artigos originais, com delineamento transversal, que envolveram amostras de crianças, adolescentes e adultos brasileiros. Nestes, foram recuperadas 14 análises, onde apenas no artigo de De Vitta et al. ${ }^{17}$ observou-se chance aumentada de DL no grupo dos adolescentes envolvidos em práticas esportivas competitivas fora da escola.

A presente evidência contrasta com referências prévias internacionais, que apontam um efeito protetor da AF à DL, como apresentado na meta-análise realizada em 36 estudos prospectivos de coorte ${ }^{15}$, onde concluiu-se que a $\mathrm{AF}$ de lazer pode reduzir o risco de
Tabela 3 - Síntese de resultados dos artigos incluídos na revisão sistemática sobre a relação entre atividade física e dor lombar $(\mathrm{n}=11)$.

\begin{tabular}{|c|c|}
\hline Grupo de referência: ativos & Grupo de referência: inativos \\
\hline \multicolumn{2}{|c|}{ Crianças e adolescentes } \\
\hline \multicolumn{2}{|c|}{ Regressão Logística } \\
\hline $\begin{array}{l}\text { Silva et al. }^{20} \\
\text { Inativos }_{\text {(analise bivariada) }} \\
\mathrm{OR}=0,93 \text { (IC95\%: 0,69-1,26) }\end{array}$ & $\begin{array}{l}\text { De Vitta et al. }{ }^{17} \\
\text { Crianças envolvidas em práticas } \\
\text { esportivas fora da escola (nâo são } \\
\text { descritas as variáveis ajustadas) }\end{array}$ \\
\hline & $\mathrm{OR}=2,58(\mathrm{IC} 95 \%: 1,92-3,48)$ \\
\hline \multicolumn{2}{|c|}{ Regressão de Poisson } \\
\hline $\begin{array}{l}\text { Lemos et al. }^{18} \\
\text { Não-praticantes de exercício físico (Uni) } \\
\text { RP = 1,03 (IC95\%: 0,97-1,08) }\end{array}$ & $\begin{array}{l}\text { Onofrio et al. }{ }^{19} \\
\text { Ativos (Uni) } \\
\mathrm{RP}=0,9 \text { (IC95\%: 0,6-1,2) } \\
\text { Deslocam-se por meio de } \\
\text { transporte motorizado para a } \\
\text { escola }^{(1)} \\
\mathrm{RP}=0,7 \text { (IC95\%: 0,5-1,0) }\end{array}$ \\
\hline \multicolumn{2}{|c|}{ Adultos } \\
\hline \multicolumn{2}{|c|}{ Regressão Logística } \\
\hline $\begin{array}{l}\text { Andrusaitis et al. }{ }^{22} \\
\text { Sem diferenças entre os grupos } \\
\text { estratificados por níveis de AF (descrito } \\
\text { na forma de texto) }\end{array}$ & $\begin{array}{l}\text { Almeida et al. }{ }^{21} \\
\text { AF moderada } \\
\text { ORni) } \\
\text { AF vigorosa (Uni) } \\
\text { OR }=1,37 \text { (IC95\%: 0,38-4,87) }\end{array}$ \\
\hline $\begin{array}{l}\text { Zanuto et al. } .^{27} \\
<180 \mathrm{~min} / \mathrm{sem} \text { de AF de lazer }{ }^{(2)} \\
\mathrm{OR}=1,65(\mathrm{IC} 95 \%: 0,66-4,09)\end{array}$ & $\begin{array}{l}\text { Silva et al. }{ }^{26} \\
\text { Praticam AF (Uni) } \\
\text { OR = 1,18 (IC95\%: 0,85-1,65) }\end{array}$ \\
\hline \multicolumn{2}{|c|}{ Regressão de Poisson } \\
\hline $\begin{array}{l}\text { Ferreira et al. }{ }^{23} \\
\text { Insuficientemente ativos em todos os } \\
\text { domínios (não são descritas as variáveis } \\
\text { ajustadas) } \\
\mathrm{RP}=1,06 \text { (IC95\%: 0,84-1,34) } \\
\text { Insuficientemente ativos no lazer (não } \\
\text { são descritas as varíaveis ajustadas) } \\
\mathrm{RP}=0,96 \text { (IC95\%: } 0,86-1,07 \text { ) }\end{array}$ & $\begin{array}{l}\text { Santana et al. }{ }^{25} \\
\text { DL em inativos }{ }^{(3)} \\
\text { RP = 1,00 (IC95\%: 0,56-1,78) }\end{array}$ \\
\hline $\begin{array}{l}\text { Meucci et al. }{ }^{24} \\
\text { Insuficientemente ativos }{ }^{(4)} \\
\mathrm{RP}=1,02 \text { (IC95\%: 0,74-1,43) }\end{array}$ & \\
\hline
\end{tabular}

(1) = Ajustada por cor da pele, idade, gênero, nível econômico, meios de transporte para a escola, conforto da cadeira escolar, exposição diária à televisão e computador; $(2)$ = Ajustada por variáveis socioeconômicas, comportamentais e excesso de peso; (3) = Ajustada pelos fatores demográficos, comportamentos em saúde, hipertensão arterial, dislipidemia, depressão, doenças no coração, percepção da saúde, percepção de estresse; (4) = Ajustada por sexo, idade, cor da pele, estado civil, grau de educação, hábito tabagista, IMC, atividade física suficiente, movimentos repetitivos no trabalho e trabalho com levantamento de peso; $\mathrm{AF}$ = atividade física; $\mathrm{IC} 95 \%$ = intervalo de confiança de 95\%; DL = dor lombar; $\mathrm{OR}=$ odds ratio; $\mathrm{RP}=$ razão de prevalências; $(\mathrm{Uni})=$ análise univariada.

DL crônica entre 11\% e 16\%. Em outra recente meta-análise, com objetivo de avaliar o efeito do exercício físico na prevenção de DL em intervenções de base populacional, foram constatadas, para além da redução do risco de DL, uma menor gravidade do seu quadro em pessoas que realizavam exercícios regularmente ${ }^{16}$. Cabe 
mencionar, novamente, que ambas as revisões não tiveram incluídos estudos brasileiros em suas sínteses.

Em uma outra revisão sistemática, que teve como objetivo investigar a eficácia de intervenções para prevenção de DL em adultos, concluiu-se sobre a eficácia do exercício (sozinho ou em combinação com estratégias educativas) na prevenção da dor $\operatorname{lombar}^{28} \mathrm{e}$, em outro estudo direcionado aos adultos, aponta-se que a inatividade física está associada a maiores alterações estruturais na coluna e incapacidade, em comparação com indivíduos considerados fisicamente ativos ${ }^{29}$.

Sobre a evidência de chances aumentadas de DL no grupo dos adolescentes envolvidos em práticas esportivas competitivas fora da escola ${ }^{17}$, uma possível justificativa para tal encontra-se na relação em forma de "U" ${ }^{30}$, onde em uma ponta da curva estão os participantes fisicamente inativos e na outra ponta estão pessoas que praticam AF de maneira excessiva; ou seja, os extremos do padrão de AF podem representar maiores riscos à DL. Este foi o único dado incluído na síntese que apontou para uma associação estatisticamente significativa. Cabe apontar, também que a literatura também sugere prevalências elevadas de DL em atletas adolescentes ${ }^{31}$.

Em outra revisão sistemática ${ }^{32}$ concluiu-se que, a ocorrência de DL está associada à natureza e à intensidade das $\mathrm{AF}$, e que existe uma carência de estudos com foco nas AF habituais diárias e sua associação com a DL. Nesse sentido, diante da alta heterogeneidade quanto à avaliação e forma de classificação da AF nas pesquisas que fundamentaram a presente síntese, sugere-se que instrumentos padronizados sejam utilizados nos futuros estudos, a fim de se verificar quais possíveis tipos e domínios de AF podem estar associados enquanto proteção ou mesmo risco ao ocasionamento e/ ou intensificação da DL.

A grande variação observada no que diz respeito aos períodos dos instrumentos para determinação das prevalências corrobora a evidência de outras revisões prévias $^{15,33-35}$. Complementarmente, a presente síntese também aponta para o fato de que boa parcela dos estudos incluídos não distinguiram, em sua avaliação, a DL como aguda ou crônica, e entre aqueles que distinguiram foi observada variabilidade na classificação.

O comportamento e o prognóstico de uma pessoa com sintoma de DL aguda é diferente do que se apresenta na DL crônica, que geralmente acarreta em comprometimento das atividades diárias ${ }^{36-37}$. Existe certa divergência na literatura sobre as durações da DL aguda e crônica, e nota-se também um grande número de pessoas que relatam DL recorrente, que difere de ambas, o que dificulta a distinção entre os eventos ${ }^{8}$. Nesse sentido, a presente evidência aponta para a importância de se buscar classificação das pessoas quanto o tipo de DL, no sentido, também, de se esclarecer melhor o possível papel da AF em relação diferentes tipos de DL.

Como limitações da pesquisa, é importante destacar que na presente síntese, todos os estudos disponíveis apresentaram delineamento transversal, que por sua vez, limitam quaisquer inferências sobre causa e efeito (suscitando, inclusive, causalidade reversa), devido ao fato de a exposição e o desfecho serem coletados em um único momento no tempo ${ }^{38}$. Outra limitação observada são as os autorrelatos, utilizados em todos os artigos investigados para avaliar tanto o nível de $\mathrm{AF}$, quanto a presença da DL na presente síntese. $\mathrm{O}$ uso de questionários é propenso ao viés de recordatório, principalmente onde os pontos de corte são longos e as medidas subjetivas do nível de $\mathrm{AF}$ estão propensas à superestimação ${ }^{39}$.

Apesar de muitas diretrizes internacionais endossarem a $\mathrm{AF}$ enquanto componente importante na prevenção e/ou tratamento da $\mathrm{DL}^{36}$, um longo percurso ainda há de ser traçado para que sejam estabelecidas suas doses e intensidades. Logo, em complemento aos tipos e domínios da AF, aponta-se a necessidade de um maior debate quanto os importantes elementos da prática de $\mathrm{AF}$, como dosagem, intensidade, adesão, postura e padrões de movimento. Possivelmente, por meio deste aprofundamento, novas aproximações entre as variáveis possam ser encontradas.

Por fim, mesmo que a maior parte dos artigos incluídos não tenha apresentado associações significativas entre as variáveis de interesse, a presente síntese aponta para a necessidade de importantes avanços metodológicos nos futuros estudos: como a necessidade da realização de estudos longitudinais, a investigação de possíveis relações entre distintos domínios da $\mathrm{AF}$ e diferentes tipos de DL, a necessidade de padronização nos pontos de corte utilizados pelos instrumentos para determinar AF suficiente e a prevalência da DL e utilização de medidas objetivas, em paralelo ao questionário, para avaliação da AF.

\section{Conflito de interesses}

Os autores declaram não haver conflito de interesses. 


\section{Contribuição dos autores}

Polli GR, avaliação dos textos integrais e extração dos dados; conteúdo crítico para construção e desenvolvimento do texto. Falqueto H, avaliação dos títulos e resumos; conteúdo crítico para construção e desenvolvimento do texto. Czarnobai I, avaliação dos títulos e resumos; conteúdo crítico para construção e desenvolvimento do texto. Christofaro DGD, conteúdo crítico para construção e desenvolvimento do texto. Guerra PH, concepção da ideia original; montagem das buscas sistemáticas; avaliação dos títulos, resumos, textos integrais e extração dos dados; conteúdo crítico para construção e desenvolvimento do texto.

\section{Referências}

1. World Health Organization. Global Recommendations on Physical Activity for Health. Geneva: World Health Organization; 2010.

2. Kesaniemi YK, Danforth E, Jensen MD, Kopelman PG, Lefèbvre P, Reeder B. Dose-response issues concerning physical activity and health: an evidence-based symposium. Med Sci Sports Exerc. 2001;33(6):S351-8.

3. Mendonça T, Ito R. Risco cardiovascular, aptidão física e prática de atividade física de idosos de um parque de São Paulo. Rev Bras Ci Mov. 2004;12(2):19-24.

4. Ouriques EPM, Fernandes JA. Atividade fisica na terceira idade: uma forma de prevenir a osteoporose? Rev Bras Ativ Fis Saúde. 1997;2(1):53-9.

5. Murray CJL, Barber RM, Foreman KJ, Ozgoren AA, AbdAllah F, Abera SF, et al. Global, regional, and national disability-adjusted life years (DALYs) for 306 diseases and injuries and healthy life expectancy (HALE) for 188 countries, 1990-2013: quantifying the epidemiological transition. Lancet. 2015;386(10009):2145-91.

6. Woolf AD, Pfleger B. Burden of major musculoskeletal conditions. Bull World Health Organ. 2003;81(9):646-56.

7. Sociedade Brasileira de Reumatologia, Comitê de Coluna Vertebral. Consenso Brasileiro sobre Lombalgias e Lombociatalgias. São Paulo: Sociedade Brasileira de Reumatologia; 2000

8. Airaksinen O, Brox JI, Cedraschi C, Hildebrandt J, KlaberMoffett J, Kovacs F, et al. Chapter 4: European guidelines for the management of chronic nonspecific low back pain. Eur Spine J. 2006;15(Suppl 2):192-300.

9. Hoy D, Bain C, Williams G, March L, Brooks P, Blyth F, et al. A systematic review of the global prevalence of low back pain. Arthritis Rheum. 2012;64(6):2028-37.

10. Lemeunier N, Leboeuf-Yde C, Gagey O. The natural course of low back pain: a systematic critical literature review. Chiropr Man Therap. 2012;20(1):33.

11. Hestbaek L, Leboeuf-Yde C, Kyvik KO, Manniche C. The course of low back pain from adolescence to adulthood. Spine. 2006;31(4):468-72.

12. Global Burden of Disease Study 2013 Collaborators T, Barber RM, Bell B, Bertozzi-Villa A, Biryukov S, Bolliger I, et al. Global, regional, and national incidence, prevalence, and years lived with disability for 301 acute and chronic diseases and injuries in 188 countries, 1990-2013: A systematic analysis for the Global Burden of Disease Study 2013. Lancet. 2015;386(9995):743-800.

13. Meziat Filho N, Silva GA. Invalidez por dor nas costas entre segurados da Previdência Social do Brasil. Rev Saude Pública.
2011;45(3):494-502.

14. Björck-van Dijken C, Fjellman-Wiklund A, Hildingsson C. Low back pain, lifestyle factors and physical activity: A population-based study. J Rehabil Med. 2008;40(10):864-9.

15. Shiri R, Falah-Hassani K. Does leisure time physical activity protect against low back pain? Systematic review and metaanalysis of 36 prospective cohort studies. Br J Sports Med. 2017;51(19):1410-8.

16. Shiri R, Coggon D, Falah-Hassani K. Exercise for the prevention of low back pain: systematic review and meta-analysis of controlled trials. Am J Epidemiol. 2017;187(5):1093-1101.

17. De Vitta A, Martinez MG, Piza NT, Simeão SFAP, Ferreira NP. Prevalência e fatores associados à dor lombar em escolares. Cad Saude Pública. 2011;27(8):1520-8.

18. Lemos AT, Santos FR, Moreira RB, Machado DT, Braga FCC, Gaya ACA. Ocorrencia de dor lombar e fatores associados em crianças e adolescentes de uma escola privada do sul do Brasil. Cad Saúde Pública. 2013;29(11):2177-85.

19. Onofrio AC, Silva MC, Domingues MR, Rombaldi AJ. Acute low back pain in high school adolescents in Southern Brazil: Prevalence and associated factors. Eur Spine J. 2012;21(7):1234-40.

20. Silva GRR, Pitangui ACR, Xavier MKA, Correia-Júnior MAV, De Araújo RC. Prevalence of musculoskeletal pain in adolescents and association with computer and videogame use. J Pediatr. 2016;92(2):188-96.

21. Almeida ICGB, Sá KN, Silva M, Baptista A, Matos MA, Lessa Í. Prevalência de dor lombar crônica na população da cidade de Salvador. Rev Bras Ortop. 2008;43(3):96-102.

22. Andrusaitis SF, Oliveira RP, Barros Filho TEP. Study of the prevalence and risk factors for low back pain in truck drivers in the state of São Paulo, Brazil. Clinics. 2006;61(6):503-23.

23. Ferreira GD, Silva MC, Rombaldi AJ, Wrege ED, Siqueira F V, Hallal PC, et al. Prevalência de dor nas costas e fatores associados em adultos do Sul do Brasil: estudo de base populacional. Rev Bras Fisioter. 2011;15(1):31-6.

24. Meucci RD, Fassa AG, Paniz VM V, Silva MC, Wegman DH. Increase of chronic low back pain prevalence in a medium-sized city of southern Brazil. BMC Musculoskelet Disord. 2013;14(2):360-6.

25. Santana JDO,Peixoto SV.Inatividade física e comportamentos adversos para a saúde entre professores universitários. Rev Bras Med Esporte. 2017;23(2):103-8.

26. Silva MC, Fassa AG, Valle NC. Chronic low back pain in a Southern Brazilian adult population: prevalence and associated factors. Cad Saúde Pública. 2004;20(2):377-85.

27. Zanuto EAC, Codogno JS, Christófaro DGD, Vanderlei LCM, Cardoso JR, Fernandes RA. Prevalence of low back pain and associated factors in adults from a middle-size Brazilian city. Cien Saúde Colet. 2015;20(5):1575-82.

28. Steffens D, Maher CG, Pereira LSM, Stevens ML, Oliveira VC, Chapple M, et al. Prevention of low back pain. JAMA Intern Med. 2016;176(2):199-208.

29. Teichtahl AJ, Urquhart DM, Wang Y, Wluka AE, O'Sullivan $\mathrm{R}$, Jones $\mathrm{G}$, et al. Physical inactivity is associated with narrower lumbar intervertebral discs, high fat content of paraspinal muscles and low back pain and disability. Arthritis Res Ther. 2015;17(1):114.

30. Heneweer H, Vanhees L, Picavet SJH. Physical activity and low back pain: A U-shaped relation? Pain. 2009;143(1):21-5.

31. De Luigi AJ. Low Back Pain in the Adolescent Athlete. Phys Med Rehabil Clin N Am. 2014;25(4):763-88. 
32. Heneweer H, Staes F, Aufdemkampe G, van Rijn M, Vanhees L. Physical activity and low back pain: a systematic review of recent literature. Eur Spine J. 2011;20(6):826-45.

33. Sitthipornvorakul E, Janwantanakul P, Purepong N, Pensri $\mathrm{P}$, Van der Beek AJ. The association between physical activity and neck and low back pain: a systematic review. Eur Spine J. 2011;20(5):677-89.

34. Hendrick P,Milosavljevic S, Hale L, Hurley DA,McDonough $\mathrm{S}$, Ryan B, et al. The relationship between physical activity and low back pain outcomes: a systematic review of observational studies. Eur Spine J. 2011;20(3):464-74.

35. Loney PL, Stratford PW. The Prevalence of Low Back Pain in Adults: A Methodological Review of the Literature. Phys Ther. 1999;79(4):384-96.

36. Helfenstein Junior M, Goldenfum MA, Siena C. Lombalgia ocupacional. Rev Assoc Med Bras. 2010;56(5):583-9.

37. Reikerås O, Storheim K, Holm I, Friis A, Brox JI. Disability, Pain, Psychological Factors And Physical Performance In Healthy Controls, Patients With Sub-Acute And Chronic Low Back Pain: A Case-Control Study. J Rehabil Med.
2005;37(2):95-9.

38. Bastos JLD, Duquia RP. Um dos delineamentos mais empregados em epidemiologia: Estudo transversal. Sci Med. 2013;23(2):229-32.

39. Verbunt JA, Huijnen IPJ, Köke A. Assessment of physical activity in daily life in patients with musculoskeletal pain. Eur J Pain. 2009;13(3):231-42.

40. Bastos JP, Araujo CL, Hallal PC. Prevalence of insufficient physical activity and associated factors in Brazilian adolescents. J Phys Act Health. 2008;5(6):777-94.

41. Vidal AR. Dor lombar inespecífica em alunos adolescentes em função do género, idade e nível de actividade física [dissertação de mestrado]. Porto: Universidade do Porto;2009.

42. Kuorinka I, Jonsson B, Kilbom A, Vinterberg H, BieringSørensen F, Andersson G, et al. Standardised Nordic questionnaires for the analysis of musculoskeletal symptoms. Appl Ergon. 1987;18(3):233-7.

Recebido: $11 / 09 / 2018$

Aprovado: 20/01/2019

\section{Como citar este artigo:}

Polli GR, Falqueto H, Czarnobai I, Christofaro DGD, Guerra PH. Atividade física e dor lombar em brasileiros: uma revisão sistemática. Rev Bras Ativ Fís Saúde. 2018;23:e0047. DOI: 10.12820/rbafs.23e0047 